OPEN ACCESS

Edited by:

Dennis Qing Wang,

Southern Medical University, China

Reviewed by:

Raghavan Pillai Raju,

Augusta University, United States

Shan Ping Yu,

Emory University, United States

*Correspondence:

Guo-Yuan Yang gyyang0626@163.com

Kunlin Jin

kunlin.jin@unthsc.edu

Specialty section:

This article was submitted to

Non-Neuronal Cells,

a section of the journal

Frontiers in Cellular Neuroscience

Received: 17 July 2020 Accepted: 24 August 2020

Published: 30 October 2020

Citation:

Wang J, Wang B, Jiang L, Zhou K, Yang G-Y and Jin K (2020) The Effect of IDO on Neural Progenitor Cell Survival Under Oxygen Glucose Deprivation.

Front. Cell. Neurosci. 14:581861. doi: 10.3389/fncel.2020.581861

\section{The Effect of IDO on Neural Progenitor Cell Survival Under Oxygen Glucose Deprivation}

\author{
Jixian Wang ${ }^{1}$, Brian Wang ${ }^{2}$, Lei Jiang ${ }^{2}$, Kaijing Zhou ${ }^{2}$, Guo-Yuan Yang ${ }^{3,4 *}$ and Kunlin Jin ${ }^{2 *}$ \\ ${ }^{1}$ Department of Rehabilitation, Ruijin Hospital, Shanghai Jiao Tong University School of Medicine, Shanghai, China, \\ ${ }^{2}$ Department of Pharmacology and Neuroscience, University of North Texas Health Science Center Fort Worth, Fort Worth, \\ TX, United States, ${ }^{3}$ Med-X Research Institute and School of Biomedical Engineering, Shanghai Jiao Tong University School of \\ Medicine, Shanghai, China, ${ }^{4}$ Department of Neurology, Ruijin Hospital, Shanghai Jiao Tong University School of Medicine, \\ Shanghai, China
}

Objective: Indoleamine 2,3-dioxygenase (IDO) activity plays an important role in many neurological disorders in the central nervous system, which may be associated with immunomodulation or anti-inflammatory activity. However, the action of IDO in the ischemic condition is still poorly understood. The purpose of the present study is to explore the expression and action of IDO in stem cell culture under oxygen and glucose deprivation.

Methods: Neural progenitor cells were obtained from the human embryonic stem cell line BG01. These cells underwent oxygen and glucose deprivation. We examined the IDO expression at 3 and $8 \mathrm{~h}$ of oxygen and glucose deprivation and then examined neuronal progenitor cell viability in the normal and oxygen and glucose deprivation condition using the [3-(4,5-dimethylthiazol-2-yl)-2,5-diphenyltetrazolium bromide] assay. In addition, we studied the effect of IDO inhibition and the expression of TNF- $\alpha$, IGF-1, VEGF, IL-6, FGF $\beta$, TGF $\beta$, EGF, and Leptin to explore the mechanism of IDO under the oxygen and glucose deprivation.

Results: IDO expression in neural progenitor cells increased under oxygen and glucose deprivation, which is closely associated with cell death $(p<0.05)$. Inhibiting IDO did not affect cell survival in normal neural progenitor cells. However, inhibiting IDO could attenuate cell viability under oxygen and glucose deprivation $(p<0.05)$. Further study demonstrated that IDO expression was closely associated to the growth factor's leptin expression.

Conclusions: Our results demonstrated that an increase of IDO under oxygen and glucose deprivation was associated with cell death, suggesting that inhibiting IDO could be a target for neuroprotection.

Keywords: IDO, neural progenitor cells, oxygen glucose deprivation, cell viability, leptin

Abbreviations: EGF, epidermal growth factor; 1-MT, 1-Methyltryptophan; FGF- $\beta$, fibroblast growth factor; IDO, indoleamine 2,3-dioxygenase; IGF-1, insulin-like growth factor 1; IL-6, interleukin-6; MTT, [3-(4,5-dimethylthiazol-2-yl)2,5-diphenyltetrazolium bromide; NPCs, neural progenitor cells; OGD, oxygen glucose deprivation; TGF- $\beta$, transforming growth factor; TNF- $\alpha$, tumor necrosis factor $\alpha$; VEGF, vascular endothelial growth factor. 


\section{INTRODUCTION}

Ischemic stroke is one of the leading causes of death and adult disability worldwide, which leads to massive cell death and complex pathological changes (Tang et al., 2016; Naghavi et al., 2017; Wang et al., 2017). With the recent improvement of acute ischemic stroke management, tPA induced thrombolysis and intravascular thrombectomy are likely to provide insights to develop therapeutic strategies that may benefit stroke patients (Yang et al., 2019). However, stroke morbidity and mortality are still high. To understand the mechanisms of stroke occurrence and development is extremely critical to reduce the imminent danger of stroke. Increased evidence indicated immunomodulation and inflammation is crucial in the physiopathological development of stroke (Jayaraj et al., 2019). Our previous studies demonstrated that during an ischemic stroke attack, resident microglia/macrophages were activated, which could increase cytokines such as IL$1 \beta$, IL-6, TNF $\alpha$, chemokines CXC, CC, CX3C, and XC, and adhesion molecules, calcium-independent integrins, and calcium-dependent cadherins expression in the ischemic region as well as system blood circulation (Ma et al., 2017). In addition, activated macrophages/microglia also triggered the immune system cascade through activating regulatory $\mathrm{T}$ cells, $\mathrm{T}$ cells, B cells, and the complement system (Wang et al., 2015). However, we do not know how these inflammatory or immune responses reflect the progression of these events during ischemic brain injury. A recent study in a mouse model of middle cerebral artery occlusion demonstrated that the macrophages/microglia could enhance indoleamine 2,3-dioxygenase 1 (IDO1)-dependent neurotoxic kynurenine metabolism during ischemic pathogenesis, which was closely related to the post-stroke depression (Koo et al., 2018). However, the role of IDO in the cell death under oxygen and glucose deprivation is largely unknown.

The kynurenine pathway is initiated by the oxidative metabolism of tryptophan. It has been studied since the early 20th century. No specific neurobiological activity was founded for kynurenine and its catabolic products. In 1978, a study showed that several tryptophan metabolites could produce convulsions when injected directly into the brain (Lapin, 1978). In the 1980s, there was interest in the biological function of an initial enzyme IDO in the kynurenine pathway, which could convert tryptophan to kynurenine. Pfefferkorn (1984) observed that IDO was activated by interferon in the immune response to infection, inhibiting the harmful effects of infectious mediators. However, the mechanism of the anti-infective effect was caused by the depletion of tryptophan or by the accumulation of kynurenine and its downstream metabolites was still controversial (Badawy, 2017). IDO is a heme-containing enzyme expressed in a number of tissues and cells (Yamazaki et al., 1985). IDO is an important molecule of the immune system and plays a part in the natural defense against various pathogens (Yoshida and Hayaishi, 1978; Yoshida et al., 1979). IDO has a function in the response to the inflammatory response and has an immunosuppressive function to limit $\mathrm{T}$ cell function (Munn and Mellor, 2013). IDO not only plays a key role in suppressing the anti-tumor immune response in the body (Prendergast et al., 2014), but is also involved in many neurological diseases such as atherosclerosis (Cole et al., 2015), Huntington's disease (Perkins and Stone, 1983), Alzheimer's disease, multiple sclerosis (Fiala et al., 1998; Guillemin et al., 2003), and psychiatric disorders (Savitz et al., 2015; Erhardt et al., 2017). However, the effect of IDO on these neurological disorders is still debatable or just beginning to receive a small degree of attention (Schwarcz and Stone, 2017). The function of IDO during cerebral ischemia has not been well studied both in vivo and in vitro. In the present study, we examined the IDO expression in neural progenitor cells during oxygen and glucose deprivation to explore the function of IDO in the neuronal death process. We further studied the relationship between IDO expression and the inflammatory response.

\section{MATERIALS AND METHODS}

Research protocol was approved by the Institutional Animal Care and Use Committee (IACUC) of Shanghai Jiao Tong University, Shanghai, China. The experiments were performed under the ARRIVE guideline.

\section{Cell Culture and Identification}

Neural stem/progenitor cells (NPCs) derived from the human embryonic stem cells ( $h \mathrm{ESC}$ ) line BG01 were obtained from Aruna Biomedical (Athens, GA, USA). Cells were seeded on $0.01 \%$ polyornithine (Sigma-Aldrich, St. Louis, MO, USA) and $20 \mu \mathrm{g} / \mathrm{ml}$ laminin (Stemgent Inc., Cambridge, MA, USA) coated dishes and cultured in a medium, consisting of a neurobasal medium with a B27 supplement containing $2 \mathrm{mmol} / \mathrm{l}$ L-glutamine (both from Gibco Life Sciences, Grand Island, USA), $50 \mu \mathrm{g} / \mathrm{ml} \mathrm{Pen/Strep} \mathrm{(Invitrogen,} \mathrm{Carlsbad,} \mathrm{CA,} \mathrm{USA),} 10 \mathrm{ng} / \mathrm{ml}$ leukemia inhibitory factor (Thermo Fisher Scientific, Waltham, MA, USA), and $20 \mathrm{ng} / \mathrm{ml}$ fibroblast growth factor-2 (R\&D Systems, Minneapolis, MN, USA; Jin et al., 2010). Cells were cultured in a humidified incubator at $37^{\circ} \mathrm{C}$ with $5 \% \mathrm{CO}_{2}$. They were propagated in the medium and, on reaching $90 \%$ to $100 \%$ confluence, were triturated to detach them from the dish.

\section{Immunofluorescent Staining}

Cells were treated with $100 \%$ methanol (chilled at $-20^{\circ} \mathrm{C}$ ) for 5 min. Then cells were washed with ice-cold PBS. The cells were incubated with $1 \%$ BSA in PBS for 60 min to block any unspecific binding of the antibodies. Then the cells were incubated in the primary antibodies Nestin (1:200 dilution, Millipore) and SOX2 (1:100 dilution, GeneTex, Zeeland, MI, USA), respectively at $4^{\circ} \mathrm{C}$ overnight, followed by incubation with secondary antibodies (Life Technologies) for $1 \mathrm{~h}$ at room temperature. The results were observed under confocal microscopy (Zeiss, Thornwood, NY, USA). The photomicrographs were taken for cell identification.

\section{Oxygen-Glucose Deprivation (OGD)}

The procedure of oxygen-glucose deprivation (OGD) and reoxygenation was as follows: NPCs were seeded on $0.01 \%$ polyornithine (Sigma-Aldrich) and $20 \mu \mathrm{g} / \mathrm{ml}$ laminin (Stemgent) coated dishes and cultured. Cells were transferred 
from the original media to the deoxygenated glucose-free neurobasal medium. The OGD and reoxygenation experiment was performed using a specialized sealed chamber, which contained an anaerobic gas mixture $\left(95 \% \mathrm{~N}_{2}\right.$ and $\left.5 \% \mathrm{CO}_{2}\right)$ at $37^{\circ} \mathrm{C}$. NPCs cultured in the NPC culture medium without OGD were used as a negative control. After 3 or $8 \mathrm{~h}$ treatment, these cells were removed from the chamber; and the medium in the cultures was replaced with the maintenance medium. In the 8-h OGD groups, cells were then allowed to recover for $24 \mathrm{~h}$ in a regular incubator.

\section{Western Blot Analysis}

Cells were collected and lysed in RIPA (Millipore, Burlington, USA) supplemented with a cocktail (Sigma-Aldrich, St. Louis, MO, USA), $1 \mathrm{mmol} / \mathrm{l}$ PMSF (Thermo), and a phosphatase inhibitor (Thermo). For Western blots analysis, denatured samples containing the same amount of proteins $(30 \mu \mathrm{g})$ were loaded onto the resolving gel (EpiZyme, Shanghai, China) for electrophoresis. Proteins were then transferred onto a nitrocellulose membrane (Whatman, Piscataway, NJ, USA). The membrane was blocked with blocking buffer (EpiZyme) and then incubated with primary antibodies at the following dilution IDO (1:200 dilution, LifeSpan Biosciences, Seattle, WA, USA) at $4^{\circ} \mathrm{C}$ overnight, respectively. The membrane was washed, incubated with the appropriate HRP-conjugated secondary antibody for $1 \mathrm{~h}$, and then reacted with enhanced chemiluminescence substrate (Pierce, Rockford, IL, USA). The results were recorded by the Quantity One image software (Bio-Rad, Hercules, CA, USA) and relative intensity was calculated using the Image software (NIH, USA).

\section{MTT Assay}

Cell survival assays were performed using an MTT kit (Sigma-Aldrich, St. Louis, MO, USA). The MTT solution was added to each well and incubated at $37^{\circ} \mathrm{C}$ for $4 \mathrm{~h}$. After incubation, MTT solvent was added into each well. The plate was wrapped in foil and shaken on an orbital shaker for $15 \mathrm{~min}$. Occasionally, pipetting of the liquid was required to fully dissolve the MTT formazan. Absorbance was measured at $590 \mathrm{~nm}$ using a microplate reader (Thermo Fisher Scientific, Waltham, MA, USA).

\section{ELISA Assay}

To assess the release of angiogenesis-related cytokines in $h \mathrm{ESC}$ derived NPCs after OGD, and 1-MT treatment, the amount of released TNF- $\alpha$, IGF-1, VEGF, IL- 6 , FGF- $\beta$, TGF- $\beta$, EGF, and Leptin was measured by using a human angiogenesis ELISA kit (Signosis Inc., Santa Clara, CA, USA). The supernatant was collected from each sample. A hundred microliter sample was added into the well and incubated, for $1 \mathrm{~h}$ at room temperature with gentle shaking. Each well was washed by adding $200 \mu \mathrm{l}$ of $1 \times$ assay wash buffer three times. A total
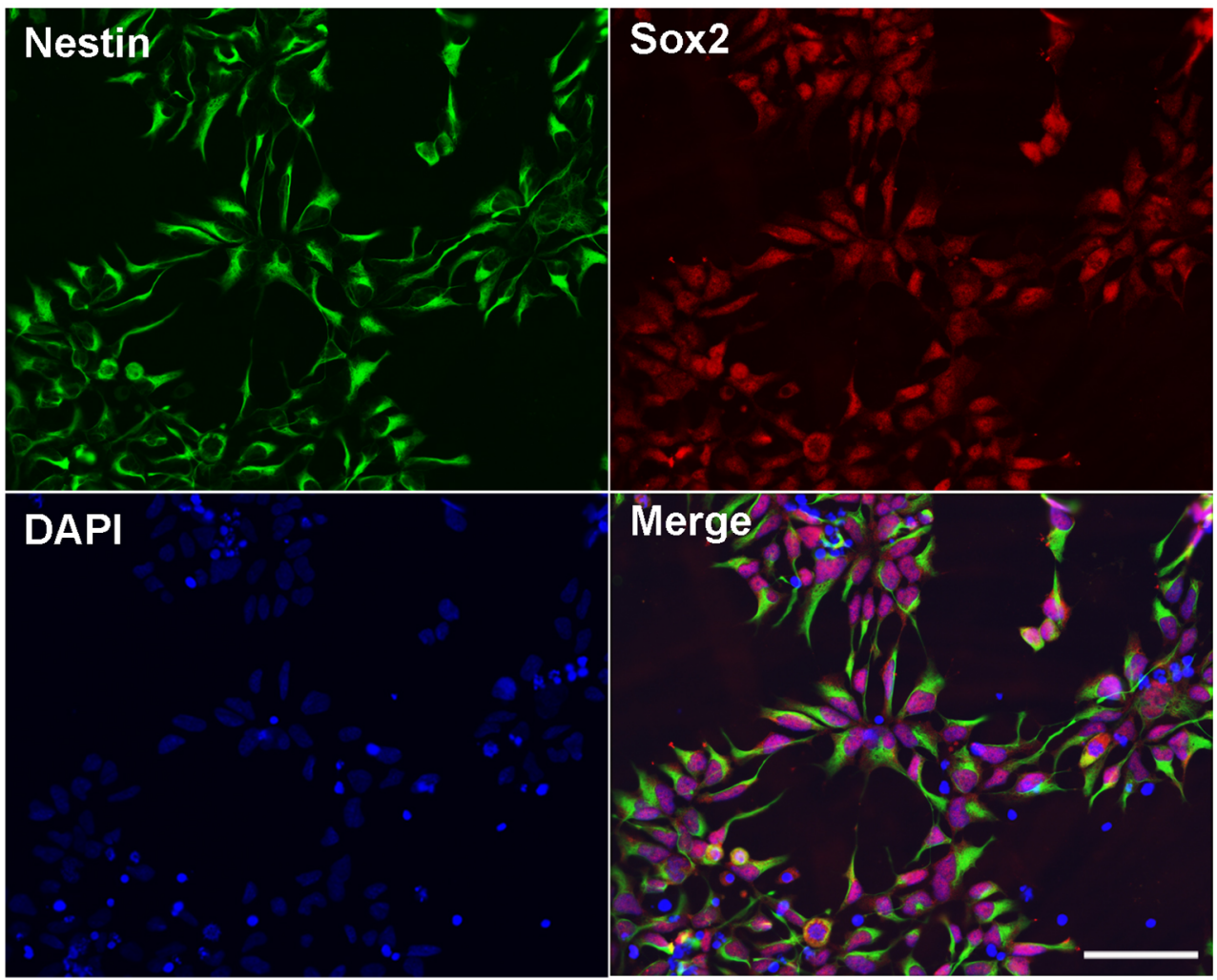

FIGURE 1 | Identification of human embryonic stem cells ( $h$ ESC)-derived neural progenitor cells (NPCs). Immunostaining images of $h$ ESC-derived NPCs showed anti-Nestin (green), Sox2 (red), and DAPI (blue). Scale bar $=50 \mu \mathrm{m}$. 
of $100 \mu \mathrm{l}$ of diluted biotin-labeled antibody mixture and 100 $\mu l$ of diluted streptavidin-HRP conjugate were in turn added to each well and incubated respectively for $1.5 \mathrm{~h}$ at room temperature with gentle shaking. A total of $100 \mu \mathrm{l}$ of substrate was added to each well and incubated for $30 \mathrm{~min}$. A total of $50 \mu \mathrm{l}$ of stop solution was added to each well. The optical density was determined by a microplate reader at $450 \mathrm{~nm}$ within $30 \mathrm{~min}$

\section{Statistical Analysis}

Quantitative data were presented as mean \pm SD and compared by one-way and two-way analysis of variance (ANOVA) with repeated measures, followed by post hoc multiple comparison tests (Fisher PLSD or Student's paired $t$-test with the Bonferroni correction) using the SPSS software (v18.0, SPSS Inc., Chicago, IL, USA). A probability value of less than 0.05 was considered to represent statistical significance.

\section{RESULTS}

\section{NPC Identification and Culture}

$h$ ESC line BG01-derived NPCs were cultured and maintained in a medium. The morphology of the NPCs were characterized by a double immunostaining with anti-Nestin and anti-Sox2. We found that more than $99 \%$ of cells expressed both Nestin and Sox2 (Figure 1), which indicated that these cells showed the NPC morphology and could be appropriately used in the subsequent experiments.

\section{IDO Expression Increased in the NPCs After OGD}

To examine the expression of IDO in the NPCs during oxygen and glucose deprivation, we performed an NPCs OGD model. Western blot results showed that IDO expression was significantly increased in NPCs after 3 and 8 h of OGD compared to the NPCs that were in a normal condition (Figure 2, $p<0.05$ ). There was the potential that IDO expression would increase with time.

\section{Inhibition of IDO Attenuated NPC Viability}

$1-\mathrm{MT}$ is an inhibitor of IDO. To explore the effect of IDO on the viability of NPCs, 1-MT was used to inhibit IDO expression. As is shown in the Figure $\mathbf{3 A}$, some of the NPCs began to die after $8 \mathrm{~h}$ of OGD. 1-MT treatment further exacerbated the damage of the NPCs. Using the MTT method, we found that 1-MT had no effect on the survival in normal NPCs (Figure 3B, left, $p>0.05$ ). However, 1-MT significantly reduced the viability of NPCs after $8 \mathrm{~h}$ of OGD at $62.5-500 \mu \mathrm{g} / \mathrm{ml}$ concentrations (Figure 3B, middle, $p<0.05$ to $p<0.001$ ). Moreover, 1-MT had the same effect on the viability of NPCs after $8 \mathrm{~h}$ of OGD and $24 \mathrm{~h}$ reperfusion (Figure 3B, left, $p<0.01)$.

\section{IDO Inhibition Reduced Cytokine Expression}

To further explore the molecular mechanism of IDO, ELISA was applied to detect cytokine expression in OGD-treated NPCs. As

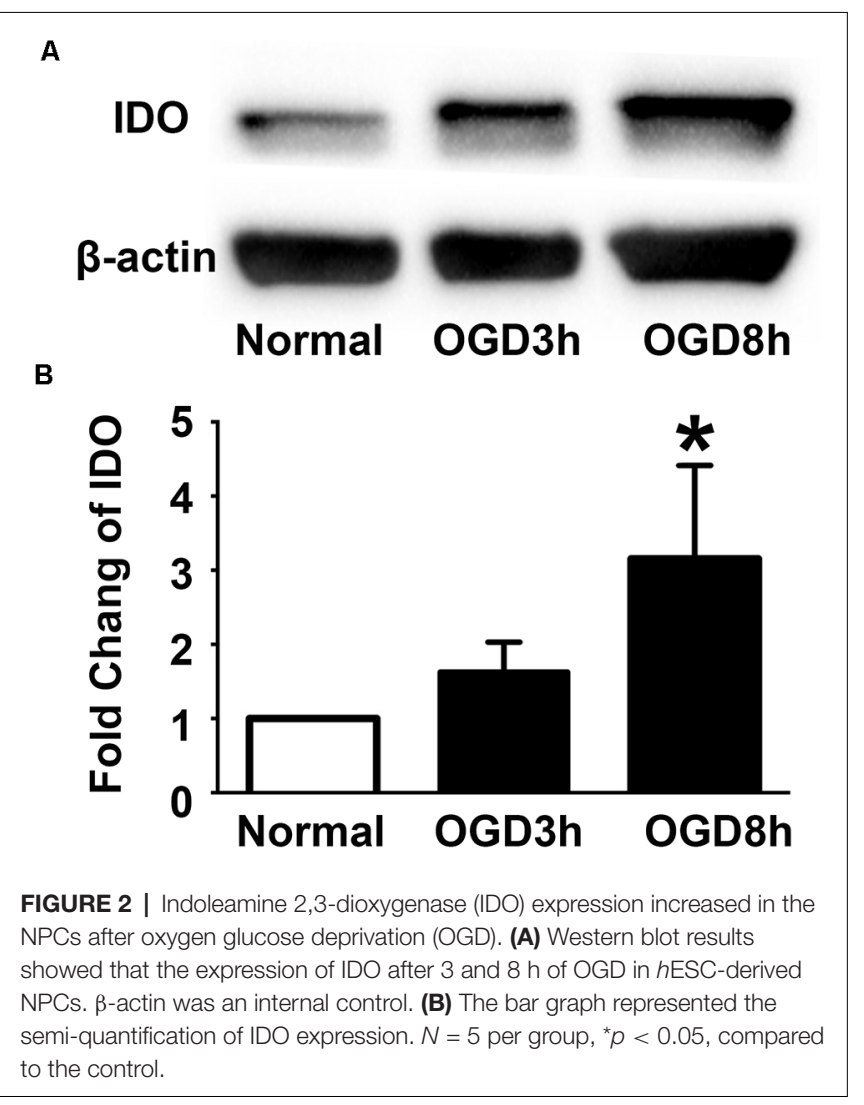

shown in Figure 4, we found that after 8 h of OGD, IGF-1, FGF- $\beta$, TGF- $\beta$, and Leptin expression in NPCs were significantly increased compared to the normal NPCs $(p<0.01)$. However, only Leptin was significantly decreased after $8 \mathrm{~h}$ of OGD with 1 -MT treatment $(p<0.05)$. This phenomenon was also observed in the group of $8 \mathrm{~h}$ of OGD and $24 \mathrm{~h}$ reperfusion with 1-MT treatment $(p<0.05)$.

\section{DISCUSSION}

Although IDO plays an important role in many neurological diseases such as Huntington's disease, Alzheimer's disease, multiple sclerosis, and psychiatric disorders, the effect of IDO in the ischemic neuronal injury is largely unknown. In the present study, we used an OGD-treated NPC-cultured model in vitro which demonstrated that: (1) the expression of IDO in NPCs increased under the OGD condition, which was closely related to cell survival; (2) the inhibition of IDO could attenuate NPC viability; and (3) increased IDO is closely associated to growth factors TNF $\alpha, \mathrm{FGF} \beta$, and Leptin expression. Our results suggested that inhibiting IDO could be a potential approach for neuroprotection in future studies in vivo.

After the discovery of IDO, the kynurenine pathway and its roles in the nervous and immune systems development was evaluated. IDO was involved not only in primarily peripheral afflictions including arthritis and atherosclerosis but also in central inflammation and immune function in Alzheimer's disease, multiple sclerosis, and Huntington's disease. These 

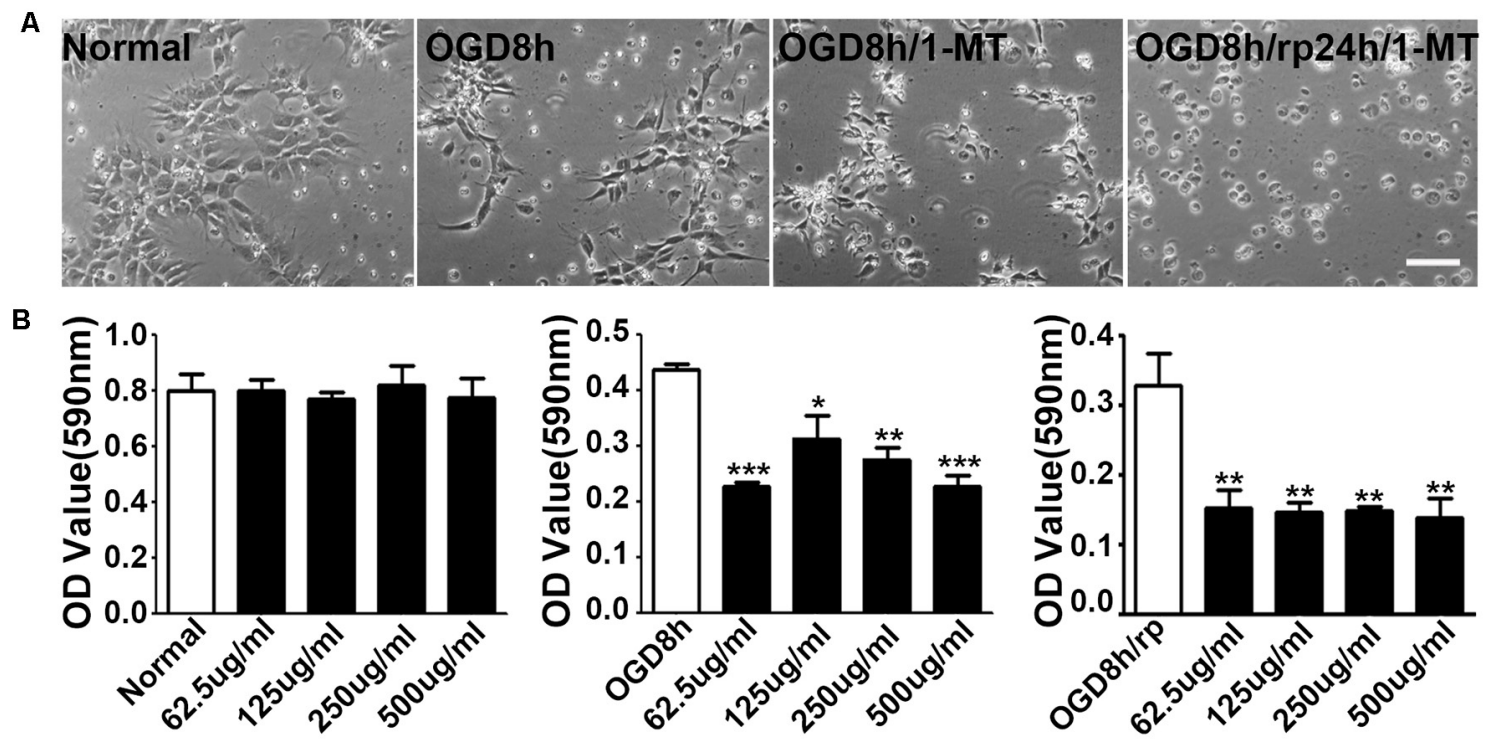

FIGURE 3 | Inhibition of IDO attenuated NPC viability. (A) Photomicrographs taken with a phase contract microscope showed neuroepithelial (NEP) morphological changes after 8-h OGD, 8-h OGD plus 1-MT treatment, and 8-h OGD followed by 24-h reperfusion plus 1-MT treatment. Scale bar = 50 $\mu$ m. (B) Bar graphs presented the MTT results: the effect of 1-DL-MT at 62.5, 125, 250, and $500 \mu \mathrm{g} / \mathrm{ml}$ on NEP cells under normal condition (left), after 8-h OGD (middle), and 8-h OGD plus 24-h reperfusion (right). $N=5$ per group, ${ }^{\star} p<0.05,{ }^{\star \star} p<0.01,{ }^{\star \star \star} p<0.01$, compared to the controls.

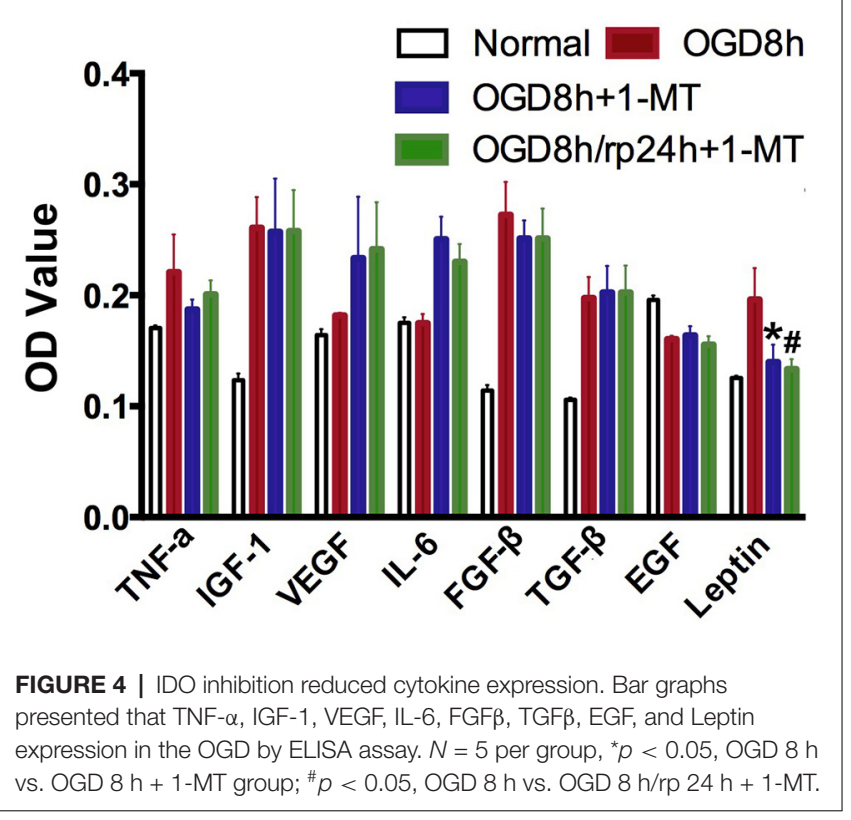

interactions could contribute to a physiologic and pathogenic role to the immune system activity with potentially common targets. IDO could be activated in astrocytes, microglia, and even in the neurons (Guillemin et al., 2003, 2005). However, if IDO was expressed in NPCs it was not well understood. An interesting topic of increasing attention is the link between kynurenines and stem cell biology. Human and mouse mesenchymal stem cells (MSCs) and neural stem cells (NSCs) expressed the kynurenine pathway (Croitoru-Lamoury et al., 2011). Pluripotent progenitor cells had IDO-dependent immunomodulatory properties, implicating kynurenine, and possibly its downstream metabolites (Jacobs et al., 2013). Numerous studies demonstrated that MSC transplantation had neuroprotection and neuronal repair function, which was a new clinical approach for repairing injured tissue. The function of MSCs could modulate immunological responses via $\mathrm{T}$ cell suppression, through IDO and toll-like receptor (TLR) signaling pathways (Lyakisheva et al., 2002; Aggarwal and Pittenger, 2005). In this study, we chose NPCs to explore the effect of IDO in brain neurons because NPCs were an important component during injured brain tissue repair. We found that, similar to the MSCs, IDO expression increased in NPCs during OGD, which is related to the cell viability. Increased IDO could attenuate NPC death during OGD, suggesting that IDO plays a neuroprotection role in the cell survival process.

In studies of the role of kynurenines in the brain, the most useful information was commonly collected from in vivo models or acutely prepared tissue slices, while freshly dissociated cells in vitro, for example, neurons, astrocytes and microglia also provided relevant information (Guillemin et al., 2007). In a mouse middle cerebral artery occlusion model, Koo et al. (2018) found that microglial IDO expression, QUIN production, and reactive oxidative species (ROS) were prominently increased in the accumbens nucleus, hippocampus, and hypothalamus, and found that these increases were related to mouse depressive-like behaviors. They demonstrated that adjunctive antidepressant aripiprazole ameliorated depressive behavior and cognitive impairment in the ischemic mice via downregulation of IDO1, HAAO, QUIN, and ROS. The 
action of IDO could be sustained for at least 9 weeks. This in vivo study provided evidence that the IDO1-dependent neurotoxic kynurenine metabolism represented a potential therapeutic target for the treatment of post-stroke dementia. However, our result was different from their study. We found that the over-expression of IDO could enhance cell viability while inhibiting IDO action could attenuate NPC survival. We believe that the effect of IDO in the early phase or the later phase of ischemia could be different. In the early stage, IDO may be involved in the acute inflammatory response while in the later stage it may promote anti-inflammation and immune-regulation. The action of IDO in the long term of neurological diseases could also be different with acute cerebral vascular disease. In addition, an argument against a special role of NMDA receptor inhibition could not be duplicated for the action of kynurenic acid in several experiments in vitro and in vivo (Hilmas et al., 2001; Beggiato et al., 2013), suggesting that IDO was involved in several signal pathways. Nevertheless, the effect of IDO on ischemic neurons and the brain should to be further studied.

Growing evidence indicated that IDO, TGF- $\beta 1$, and PGE2 may represent relevant mediators of NK cell functional inhibition (Di Nicola et al., 2002; Meisel et al., 2004; Aggarwal and Pittenger, 2005). Quantitative real-time PCR confirmed that TNF- $\alpha$ could significantly upregulate HGF mRNA (English et al., 2007). Another study neutralized HGF and TGF$\beta 1$ and found that HGF worked in synergy with TGF- $\beta 1$ to resist $\mathrm{T}$ cell recognition (Di Nicola et al., 2002). All this suggested that IDO and TGF- $\beta 1$ together regulate the immune effect of NPCs. We found that increased IDO is closely associated to the cytokine TNF- $\alpha$, and growth factors FGF- $\beta$ and Leptin, but not IGF-1, VEGF, IL-6, TGF- $\beta$, and EGF expression. This finding indicated that IDO is not an exclusive mechanism for NPC protection especially in the early stage of ischemia.

\section{REFERENCES}

Aggarwal, S., and Pittenger, M. F. (2005). Human mesenchymal stem cells modulate allogeneic immune cell responses. Blood 105, 1815-1822. doi: 10.1182/blood-2004-04-1559

Badawy, A. A.-B. (2017). Kynurenine pathway of tryptophan metabolism: regulatory and functional aspects. Int. J. Tryptophan Res. 10:1178646917691938. doi: 10.1177/1178646917691938

Beggiato, S., Antonelli, T., Tomasini, M. C., Tanganelli, S., Fuxe, K., Schwarcz, R., et al. (2013). Kynurenic acid, by targeting alpha7 nicotinic acetylcholine receptors, modulates extracellular GABA levels in the rat striatum in vivo. Eur. J. Neurosci. 37, 1470-1477. doi: 10.1111/ejn.12160

Cole, J. E., Astola, N., Cribbs, A. P., Goddard, M. E., Park, I., Green, P., et al. (2015). Indoleamine 2,3-dioxygenase- 1 is protective in atherosclerosis and its metabolites provide new opportunities for drug development. Proc. Natl. Acad. Sci. U S A 112, 13033-13038. doi: 10.1073/pnas.1517820112

Croitoru-Lamoury, J., Lamoury, F. M., Caristo, M., Suzuki, K., Walker, D., Takikawa, O., et al. (2011). Interferon-gamma regulates the proliferation and differentiation of mesenchymal stem cells via activation of indoleamine 2,3 dioxygenase (IDO). PLoS One 6:e14698. doi: 10.1371/journal.pone. 0014698

Di Nicola, M., Carlo-Stella, C., Magni, M., Milanesi, M., Longoni, P. D., Matteucci, P., et al. (2002). Human bone marrow stromal cells suppress
In conclusion, our study demonstrated that IDO expression increased in NPCs during OGD, which is associated with NPC viability. IDO may represent a potential valuable target for the treatment of injured NPCs.

\section{DATA AVAILABILITY STATEMENT}

The raw data supporting the conclusions of this article will be made available by the authors, without undue reservation.

\section{ETHICS STATEMENT}

The animal study was reviewed and approved by the Institutional Animal Care and Use Committee (IACUC) of Shanghai Jiao Tong University.

\section{AUTHOR CONTRIBUTIONS}

JW and KJ designed the experiment. JW performed all experiments, analyzed the data, and drafted the manuscript. BW, LJ, and KZ performed the Western blot and ELISA experiment. G-YY was responsible for supervising all the experimental designs, experimental performance, and finalizing the manuscript. All authors critically reviewed the manuscript. All authors contributed to the article and approved the submitted version.

\section{FUNDING}

This work was supported by the National Natural Science Foundation of China (NSFC) projects (81802232, JW; 81771251, G-YY), major projects of the Shanghai Education Commission's scientific research and innovation program (2019-01-0700-02-E00064, G-YY), and the K. C. Wong Education Foundation (G-YY).

T-lymphocyte proliferation induced by cellular or nonspecific mitogenic stimuli. Blood 99, 3838-3843. doi: 10.1182/blood.v99.10.3838

English, K., Barry, F. P., Field-Corbett, C. P., and Mahon, B. P. (2007). IFNgamma and TNF-alpha differentially regulate immunomodulation by murine mesenchymal stem cells. Immunol. Lett. 110, 91-100. doi: 10.1016/j.imlet.2007. 04.001

Erhardt, S., Schwieler, L., Imbeault, S., and Engberg, G. (2017). The kynurenine pathway in schizophrenia and bipolar disorder. Neuropharmacology 112 , 297-306. doi: 10.1016/j.neuropharm.2016.05.020

Fiala, M., Zhang, L., Gan, X., Sherry, B., Taub, D., Graves, M. C., et al. (1998). Amyloid-beta induces chemokine secretion and monocyte migration across a human blood-brain barrier model. Mol. Med. 4, 480-489. doi: 10.1007/BF03401753

Guillemin, G. J., Brew, B. J., Noonan, C. E., Takikawa, O., and Cullen, K. M. (2005). Indoleamine 2,3 dioxygenase and quinolinic acid immunoreactivity in Alzheimer's disease hippocampus. Neuropathol. Appl. Neurobiol. 31, 395-404. doi: 10.1111/j.1365-2990.2005.00655.x

Guillemin, G. J., Croitoru-Lamoury, J., Dormont, D., Armati, P. J., and Brew, B. J. (2003). Quinolinic acid upregulates chemokine production and chemokine receptor expression in astrocytes. Glia 41, 371-381. doi: 10.1002/glia.10175

Guillemin, G. J., Cullen, K. M., Lim, C. K., Smythe, G. A., Garner, B., Kapoor, V., et al. (2007). Characterization of the kynurenine pathway in human neurons. J. Neurosci. 27, 12884-12892. doi: 10.1523/JNEUROSCI.4101-07.2007 
Hilmas, C., Pereira, E. F., Alkondon, M., Rassoulpour, A., Schwarcz, R., and Albuquerque, E. X. (2001). The brain metabolite kynurenic acid inhibits alpha7 nicotinic receptor activity and increases non-alpha7 nicotinic receptor expression: physiopathological implications. J. Neurosci. 21, 7463-7473. doi: 10.1523/JNEUROSCI.21-19-07463.2001

Jacobs, S. A., Pinxteren, J., Roobrouck, V. D., Luyckx, A., van't Hof, W., Deans, R., et al. (2013). Human multipotent adult progenitor cells are nonimmunogenic and exert potent immunomodulatory effects on alloreactive T-cell responses. Cell Transpl. 22, 1915-1928. doi: 10.3727/096368912X657369

Jayaraj, R. L., Azimullah, S., Beiram, R., Jalal, F. Y., and Rosenberg, G. A. (2019). Neuroinflammation: friend and foe for ischemic stroke. J. Neuroinflammation 16:142. doi: 10.1186/s12974-019-1516-2

Jin, K., Mao, X., Xie, L., Galvan, V., Lai, B., Wang, Y., et al. (2010). Transplantation of human neural precursor cells in Matrigel scaffolding improves outcome from focal cerebral ischemia after delayed postischemic treatment in rats. J. Cereb. Blood Flow Metab. 30, 534-544. doi: 10.1038/jcbfm.2009.219

Koo, Y. S., Kim, H., Park, J. H., Kim, M. J., Shin, Y. I., Choi, B. T., et al. (2018). Indoleamine 2,3-dioxygenase-dependent neurotoxic kynurenine metabolism contributes to poststroke depression induced in mice by ischemic stroke along with spatial restraint stress. Oxid. Med. Cell. Longev. 2018:2413841. doi: $10.1155 / 2018 / 2413841$

Lapin, I. P. (1978). Stimulant and convulsive effects of kynurenines injected into brain ventricles in mice. J. Neural Transm. 42, 37-43. doi: 10.1007/BF01262727

Lyakisheva, A., Felda, O., Ganser, A., Schmidt, R. E., and Schubert, J. (2002). Paroxysmal nocturnal hemoglobinuria: differential gene expression of EGR-1 and TAXREB107. Exp. Hematol. 30, 18-25. doi: 10.1016/s0301-472x(01) 00763-9

Ma, Y., Wang, J., Wang, Y., and Yang, G. Y. (2017). The biphasic function of microglia in ischemic stroke. Prog. Neurobiol. 157, 247-272. doi: 10.1016/j. pneurobio.2016.01.005

Meisel, R., Zibert, A., Laryea, M., Gobel, U., Daubener, W., and Dilloo, D. (2004). Human bone marrow stromal cells inhibit allogeneic T-cell responses by indoleamine 2,3-dioxygenase-mediated tryptophan degradation. Blood 103, 4619-4621. doi: 10.1182/blood-2003-11-3909

Munn, D. H., and Mellor, A. L. (2013). Indoleamine 2,3 dioxygenase and metabolic control of immune responses. Trends Immunol. 34, 137-143. doi: 10.1016/j.it. 2012.10.001

Naghavi, M., Abajobir, A. A., Abbafati, C., Abbas, K. M., Abd-Allah, F., Abera, S. F., et al. (2017). Global, regional, and national age-sex specific mortality for 264 causes of death, 1980-2016: a systematic analysis for the global burden of disease study 2016. Lancet 390, 1151-1210. doi: 10.1016/S01406736(17)32152-9

Perkins, M. N., and Stone, T. W. (1983). Pharmacology and regional variations of quinolinic acid-evoked excitations in the rat central nervous system. J. Pharmacol. Exp. Ther. 226, 551-557.

Pfefferkorn, E. R. (1984). Interferon gamma blocks the growth of Toxoplasma gondii in human fibroblasts by inducing the host cells to degrade tryptophan. Proc. Natl. Acad. Sci. U S A 81, 908-912. doi: 10.1073/pnas. 81.3.908
Prendergast, G. C., Smith, C., Thomas, S., Mandik-Nayak, L., Laury-Kleintop, L., Metz, R., et al. (2014). Indoleamine 2,3-dioxygenase pathways of pathogenic inflammation and immune escape in cancer. Cancer Immunol. Immunother. 63, 721-735. doi: 10.1007/s00262-014-1549-4

Savitz, J., Dantzer, R., Meier, T. B., Wurfel, B. E., Victor, T. A., McIntosh, S. A., et al. (2015). Activation of the kynurenine pathway is associated with striatal volume in major depressive disorder. Psychoneuroendocrinology 62, 54-58. doi: 10.1016/j.psyneuen.2015.07.609

Schwarcz, R., and Stone, T. W. (2017). The kynurenine pathway and the brain: Challenges, controversies and promises. Neuropharmacology 112, 237-247. doi: 10.1016/j.neuropharm.2016.08.003

Tang, Y., Wang, L., Wang, J., Lin, X., Wang, Y., Jin, K., et al. (2016). Ischemiainduced Angiogenesis is attenuated in aged rats. Aging Dis. 7, 326-335. doi: 10.14336/AD.2015.1125

Wang, W., Jiang, B., Sun, H., Ru, X., Sun, D., Wang, L., et al. (2017). Prevalence, incidence, and mortality of stroke in china: results from a nationwide population-based survey of 480687 adults. Circulation 135, 759-771. doi: 10.1161/CIRCULATIONAHA.116.025250

Wang, J., Xie, L., Yang, C., Ren, C., Zhou, K., Wang, B., et al. (2015). Activated regulatory $\mathrm{T}$ cell regulates neural stem cell proliferation in the subventricular zone of normal and ischemic mouse brain through interleukin 10. Front Cell. Neurosci. 9:361. doi: 10.3389/fncel.2015.00361

Yamazaki, F., Kuroiwa, T., Takikawa, O., and Kido, R. (1985). Human indolylamine 2,3-dioxygenase. Its tissue distribution and characterization of the placental enzyme. Biochem. J. 230, 635-638. doi: 10.1042/bj23 00635

Yang, C.-S., Guo, A., Li, Y., Shi, K., Shi, F.-D., and Li, M. (2019). Dl-3-nbutylphthalide reduces neurovascular inflammation and ischemic brain injury in mice. Aging Dis. 10, 964-976. doi: 10.14336/AD.2019.0608

Yoshida, R., and Hayaishi, O. (1978). Induction of pulmonary indoleamine 2,3dioxygenase by intraperitoneal injection of bacterial lipopolysaccharide. Proc. Natl. Acad. Sci. U S A 75, 3998-4000. doi: 10.1073/pnas.75.8.3998

Yoshida, R., Urade, Y., Tokuda, M., and Hayaishi, O. (1979). Induction of indoleamine 2,3-dioxygenase in mouse lung during virus infection. Proc. Natl. Acad. Sci. U S A 76, 4084-4086. doi: 10.1073/pnas.76.8.4084

Conflict of Interest: The authors declare that the research was conducted in the absence of any commercial or financial relationships that could be construed as a potential conflict of interest.

The handling editor declared a past co-authorship with one of the authors KJ.

Copyright (c) 2020 Wang, Wang, Jiang, Zhou, Yang and Jin. This is an open-access article distributed under the terms of the Creative Commons Attribution License (CC BY). The use, distribution or reproduction in other forums is permitted, provided the original author(s) and the copyright owner(s) are credited and that the original publication in this journal is cited, in accordance with accepted academic practice. No use, distribution or reproduction is permitted which does not comply with these terms. 